\title{
Gait analysis, bone and muscle density assessment for patients undergoing total hip arthroplasty
}

\author{
Benedikt Magnússon (1), Halldór Jónsson jr (2,3), Pröstur Pétursson (1), Gígja \\ Magnúsdóttir (4), Grétar Halldórsson (4), Jan Triebel (2), Paolo Gargiulo (1,5)
}

(1) Biomedical engineering department, Reykjavik University Hospital; (2) Orthopaedic Clinic, Landspitali Hospital, Reykjavik; (3) Medical Faculty, Iceland University, Reykjavik; (4) Rehabilitation Clinic Grensás Landspitali Hospital, Reykjavik; (5) Department of Science, Education and Innovation, Landspitali University Hospital, Reykjavik, Iceland

\begin{abstract}
Total hip arthroplasty (THA) is performed with or without the use of bone cement. Facing the lack of reliable clinical guidelines on decision making whether a patient should receive THA with or without bone cement, a joint clinical and engineering approach is proposed here with the objective to assess patient recovery developing monitoring techniques based on gait analysis, measurements of bone mineral density and structural and functional changes of quadriceps muscles. A clinical trial was conducted with 36 volunteer patients that were undergoing THA surgery for the first time: 18 receiving cemented implant and 18 receiving non-cemented implant. The patients are scanned with Computer Tomographic (CT) modality prior-, immediately- and 12 months post-surgery. The CT data are further processed to segment muscles and bones for calculating bone mineral density (BMD). Quadriceps muscle density Hounsfield (HU) based value is calculated from the segmented file on healthy and operated leg before and after THA surgery. Furthermore clinical assessment is performed using gait analysis technologies such as a sensing carpet, wireless electrodes and video. Patients undergo these measurements prior-, 6 weeks post - and 52 weeks post-surgery. The preliminary results indicate computational tools and methods that are able to quantitatively analyze patient's condition pre and post-surgery: The spatial parameters such as step length and stride length increase 6 weeks post op in the patient group receiving cemented implant while the angle in the toe in/out parameter decrease in both patient groups.
\end{abstract}

Key Words: Muscle Density, Orthopaedic, Gait Analysis, Bone Mineral Density, Electromyography, Total Hip Replacement.

European Journal Translational Myology - Basic Applied Myology 2012; 22 (4): 155-160

For the treatment of advanced damages of hip joints THA is a well proven surgical procedure. The replacement of the hip joint with an artificial prosthesis has been one of the most effective and successful orthopedic interventions for many decades as it reproducibly restores function and reduces pain in formerly pathologic hip joints. It is applied for several pathologies, mainly in arthrosis, but also as a very beneficial treatment in osteonecrosis of the femoral head and femoral neck fractures. Due to the different mechanical properties of the prosthesis material and the bone tissue, a partial unloading of the periprosthetic bone occurs [11].

Currently there are two methodological options for THA - cemented or uncemented. The bone cement may cause reduction in bone density as a result of removal of normal stress from the bone, leading to weakening of the bone in that area and the fracture risk increases [1].

Bone loss is identified as one of the main reasons for loosening of the stem. Otherwise, thanks to the pressfit of the non-cemented stem achieved by surgery, the bone layers immediately adjacent to the stem are preloaded and encouraged to grow and getting stronger [13]. The non-cemented stem would be the better choice for most patients, but the question remains if the femur can handle the press-fitting and compressive stress during surgery. Controversy exists regarding the optimal method; large studies have shown different outcomes differentiating between cemented and 
Gait Analysis, bone and muscle density in THA patients

European Journal Translational Myology - Basic Applied Myology 2012; 22 (4): 155-160

\begin{tabular}{|c|c|c|}
\hline PARAMETER DESCRIPTION & Total/Left & Right \\
\hline Step Count & 76 & \\
\hline Distance & 3761,4 & \\
\hline Ambulation Time & 46,16 & \\
\hline Velocity & 81,5 & \\
\hline Cadence & 98,8 & \\
\hline Step Time Differential & 0,02 & \\
\hline Step Length Differential & 2,757 & \\
\hline Cycle Time Differential & 0,001 & \\
\hline Step Time(sec) & 0,617 & 0,597 \\
\hline Step Length $(\mathrm{cm})$ & 48,15 & 50,907 \\
\hline Cycle Time(sec) & 1,215 & 1,214 \\
\hline Stride Length $(\mathrm{cm})$ & 99,401 & 99,966 \\
\hline HH Base Support $(\mathrm{cm})$ & 14,431 & 14,453 \\
\hline Swing Time(sec) & 0,454 & 0,399 \\
\hline Stance Time(sec) & 0,761 & 0,815 \\
\hline Single Supp. Time(sec) & 0,399 & 0,454 \\
\hline Double Supp. Time(sec) & 0,361 & 0,366 \\
\hline Swing $\%$ of Cycle & 37,4 & 32,9 \\
\hline Stance $\%$ of Cycle & 62,6 & 67,1 \\
\hline Single Supp \% Cycle & 32,8 & 37,4 \\
\hline Double Supp \% Cycle & 29,7 & 30,1 \\
\hline Toe In / Out & $-8,6$ & -4 \\
\hline HeelOffon Time & 0,057 & 0,065 \\
\hline HeelOffOn Perc & 4,7 & 5,4 \\
\hline Double Supp Load Time & 0,163 & 0,163 \\
\hline Double Supp Load \%GC & 16,5 & 13,4 \\
\hline Double Supp Unload Time & 0,16 & 0,203 \\
\hline Double Supp Unload \%GC & 13,2 & 16,7 \\
\hline Stride Velocity & 81,973 & 82,54 \\
\hline Step Len Std Dev & 3,038 & 2,948 \\
\hline Step Time Std Dev & 0,025 & 0,028 \\
\hline Stride Length Std Dev & 5,291 & 4,539 \\
\hline Stride Time Std Dev & 0,042 & 0,048 \\
\hline Swing Time Std Dev & 0,02 & 0,021 \\
\hline Stance Time Std Dev & 0,038 & 0,038 \\
\hline Stride Velocity Std Dev & 6,151 & 5,697 \\
\hline Single Supp Time Std Dev & 0,021 & 0,02 \\
\hline Double Supp Time Std Dev & 0,031 & 0,033 \\
\hline Heel Off On Std Dev & 1,124 & 1,322 \\
\hline Supp Base On Std Dev & 2,799 & 2,363 \\
\hline Foot Length & 22,7 & 23,6 \\
\hline Foot Width & 7,112 & 7,517 \\
\hline
\end{tabular}

Fig 1 Gait analysis parameters measured during the clinical trial

uncemented methods [5]. Uncemented stems have to be more often revised due to periprosthetic fracture during the first 2 postoperative years than cemented stems. There is no noticeable difference in risk of infection between the outcomes of cemented vs. uncemented THA. In the decision making process between uncemented and cemented THA for the individual patient, bone and muscle quality is regularly included, when e.g. the biological age of the patient is estimated. Preoperative measurements of bone and muscle quality are not a standard today, although it is commonly accepted, that they can have decisive influence on the outcome. Individuals with low bone and muscle quality are good candidates for cemented THA due to the reduced risk for periprosthetic fractures during surgery and the first two postoperative years.

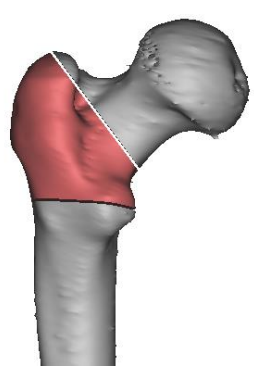

Fig 2 The $3 D$ model of the femur bone made in MIMICS. The bone has been split up to three areas. The red part (proximal femur) is the part of the bone that is used for BMD calculations.

Although age is one indicator for bone and muscle quality, individual differences due to life style and genetics are wide providing a broad range of bone density and muscle quality [10], which should be taken into consideration when deciding whether to implant a THA cemented or uncemented. Measuring both bone and muscle quality will potentially contribute to the decision making between cemented and uncemented THA.

Clinical assessment for THA surgery can be performed using Gait Analysis techniques. Different technologies have been employed to evaluate walking speed, energy consumption, range of motion (ROM), muscle function and different phases in the gait cycle. Pathological process in the hip causes changes in gait secondary to pain and reduced ROM in the hip. The total gait pattern is affected with reduced ROM, changes in muscle function, step length, walking speed, symmetry of the gait etc. After THR the gait improves but usually it is slight asymmetrical even after full recovery [12]. This can be because of leg length discrepancy, type of prosthesis, surgical approach, pain in other joints etc. Pain in the operated leg around the mid-thigh has been documented in patients with uncemented prosthesis possibly due to osteopenia [9].

In summary although many techniques are available to assess THA surgery there are no guidelines, gold standard or clear clinical recommendations currently in practice on the choice of a cemented or an uncemented THA. The present work describes a novel approach using computational tools combining measurements of bone and muscle density, gait analysis and 3-D modeling for supporting decision making in THA and selecting the optimal surgical procedure.

\section{Materials and Methods}

\section{Clinical Trial: Siral CT}

36 voluntary patients (20 females and 16 males) are enrolled into the clinical trial, 18 received cemented 
Gait Analysis, bone and muscle density in THA patients

European Journal Translational Myology - Basic Applied Myology 2012; 22 (4): 155-160

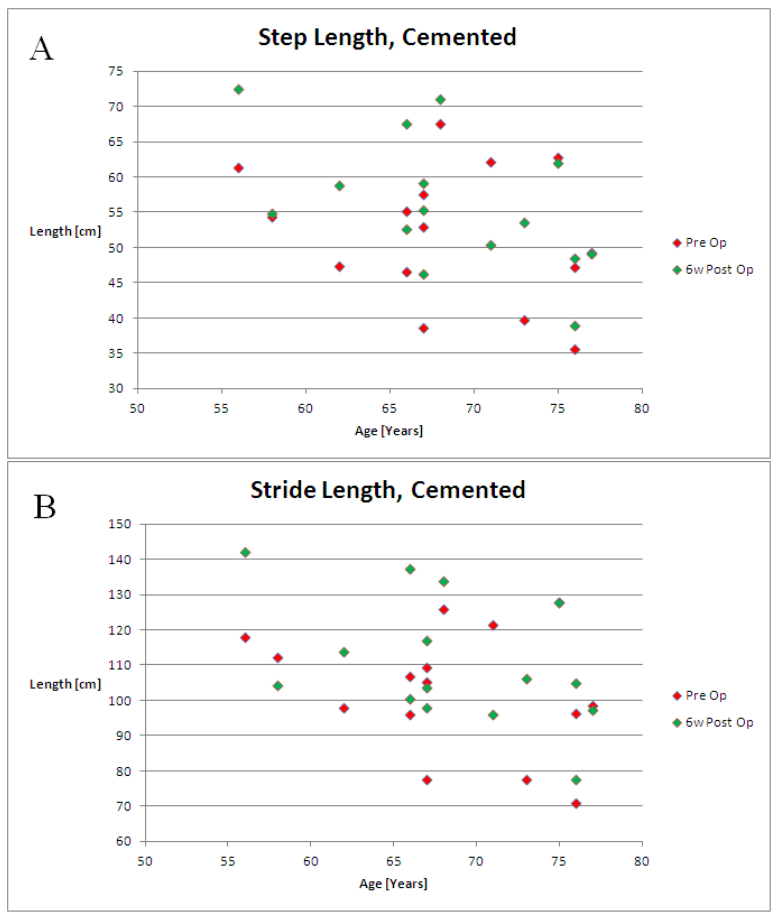

Fig 3 Gait parameters pre-and 6 weeks post-surgery. Pre-operation measurements are in red and 6 weeks post-operation measurements are in green. Both pictures display measurements from the cemented patients. Step length (A), Stride length $(B)$.

and 18 received uncemented implant. The implant type is decided according to the surgeons evaluation mostly based on patient age, gender and general clinical conditions. All patients had THA surgery for the first time. The average age at the moment of surgery is 56 for the males and 62 for the females; the youngest patient is 22 and the oldest 77 years. Patients are scanned (64 CT Philip Brilliance) before and immediately after surgery and at 52 weeks postsurgery. The scanning region starts from the iliac crest bone and ends at the middle of the femur; slices thickness is $1 \mathrm{~mm}$, slice increment is $0.5 \mathrm{~mm}$ and tube intensity is set to $120 \mathrm{KV}$. These data allow precise $3-\mathrm{D}$ reconstruction of the regions of interest.

\section{Clinical Trial: Gait Analysis}

All patients have a Gait Analysis assessment the day before surgery and at 6 and 52 weeks post-surgery. Patients walk on a sensing carpet called "GAITrite walk way system" [2] at the same time a synchronized video and EMG are taken using the wireless "Kine Measurement System" (KMS), which measures Electromyography (EMG), and digital video [6]. The GAITRite system automates measuring spatial and temporal parameters via a 14-foot electronic walkway, containing 16128 pressure switches embedded between

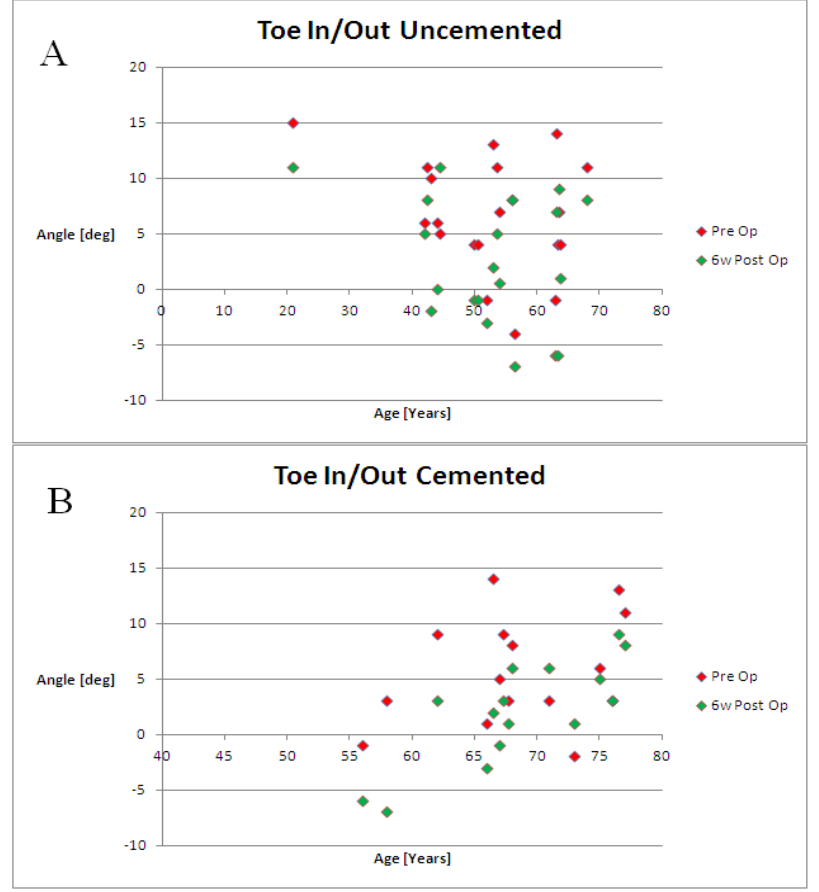

Fig 4 Gait parameters pre- and 6 weeks postsurgery. Pre-operation measurements are in red and 6 weeks post-operation measurements are in green. $4 A$ displays the measurements from the uncemented group and $4 B$ displays the measurements from the cemented group.

two sheets of vinyl. The KMS data are processed with "KinePro" [6] that is a video-based motion analysis system used to observe motion and synchronized EMG patterns during gait. LED markers are placed on the ankles, knees and hips to allow data collection on hip, knee and ankle ROM during a gait cycle. The focus is specifically on clinically important gait variables such as step length, gait symmetry, single and double support, and others parameters as seen in Figure 1.

\section{D Modeling for Bone and Muscle analysis}

The medical images coming from the CT scanner consist of grayscale information. All of these are presented in DICOM standard images imported in the "Mimics platform" [7] where femur bone and muscle are segmented from the other tissue with point- and region-based methods. The point-based method uses only the attributes of an image element for its segmentation. Thresholding is a point-based approach to image segmentation and was the technique of choice for this project. Femoral bone thresholding and segmentation is performed according to the process described in [3]. Directly from the mask properties key parameters such as volume and $\mathrm{HU}$ density are calculated from the segmented objects. 
Gait Analysis, bone and muscle density in THA patients

European Journal Translational Myology - Basic Applied Myology 2012; 22 (4): 155-160

BMD is calculated from the proximal femur in the region between femur head and lesser trochanter, along the intertrochanteric line as can be seen in Figure 2. To determine an accurate relationship between HU and BMD values, the CT scan device was calibrated with QUASAR phantom [12]. We use the linear relationship:

$B M D=H U * a 2 * H U+b+c$

where $\mathrm{a}, \mathrm{b}$ and $\mathrm{c}$ are calibration coefficients, which were calculated from the phantom data along with the corresponding statistical descriptors [4]. Voxel values of the preoperative scans concerning healthy and operated femur were converted from Hounsfield units to BMD values by using the equation:

$B M D=-8 * 10^{-9} \mathrm{HU}+0,0006 \mathrm{HU}+0,9456\left[\mathrm{~g} / \mathrm{cm}^{3}\right]$

The relation respects modality of acquisition for the scans we use in the project: 64-SCT Philips Brilliance, $120 \mathrm{KeV}$ with a correlation of $\mathrm{R} 2 \approx 0,99$.

\section{EMG analysis}

The wireless electrodes are placed on three quadriceps muscles; Vastus Lateralis, Rectus Femoris and Vastus Medialis. The patients walk six times for seven seconds each time while the EMG and video are recorded. The video is used to analyze where the patients begin their stance phase (heel strike), and where they end their stance phase (toe off). The average voltage for each muscle over this period is found in the „Kine Pro"software with an RMS filter applied so the data can be visualized better. The average from all the stance phases of each patient is then taken.

\section{Results and Discussion}

\section{Gait Analysis parameters}

The Gait Analysis technologies employed in the project allow the collection of many parameters as seen in Figure 1, the comparison of these parameters pre

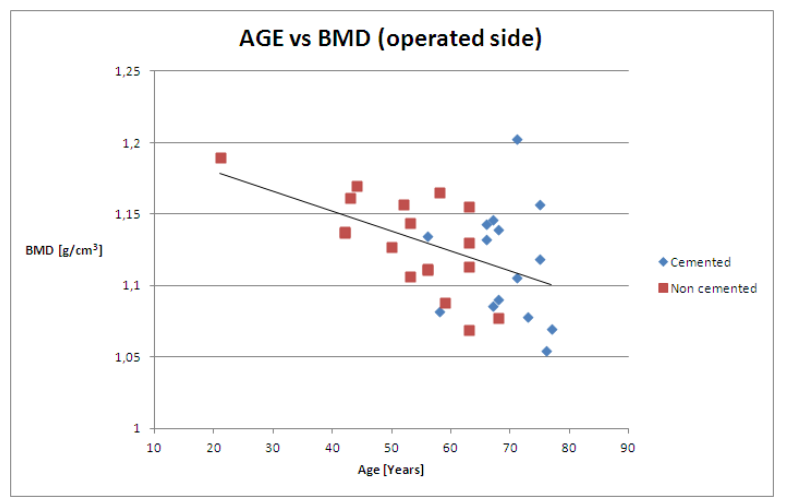

Fig 5 BMD sorted by implant technique and ordered by patient age.



Fig 6 Muscle HU based density sorted by implant technique and ordered by patient age.

and 6 weeks post THA shows some clear indications. Spatial parameters such as step length and stride length increase in most cases after 6 weeks in the patient group receiving cemented implant (Figure $3 \mathrm{~A} \& \mathrm{~B}$ ). The step length increased for 12 of 15 patients and the average increase of the step length is 4,16 centimeters. The stride length increased for 11 of 15 patients and the average increase of the stride length is 7,91 centimeters. For both patient groups an angle decrease in the toe in/out parameter (formed by the line connecting heel strike and toe - off plantar surface centers of pressure and the forward progression line) is measured 6 weeks after THA as depicted in Figure 4. The angle decreased for 25 of 35 patients. The uncemented group gets 2,2 degrees closer to the zero on average and the cemented group gets 1,8 degrees closer to the zero on average

\section{EMG, Bone and Muscles}

The pre-operative BMD measurements on the leg receiving the implant show a tendency of decreasing with the patient age as normally expected. Indeed according to the current implant policy, older patients receive cement implant because the bones here are

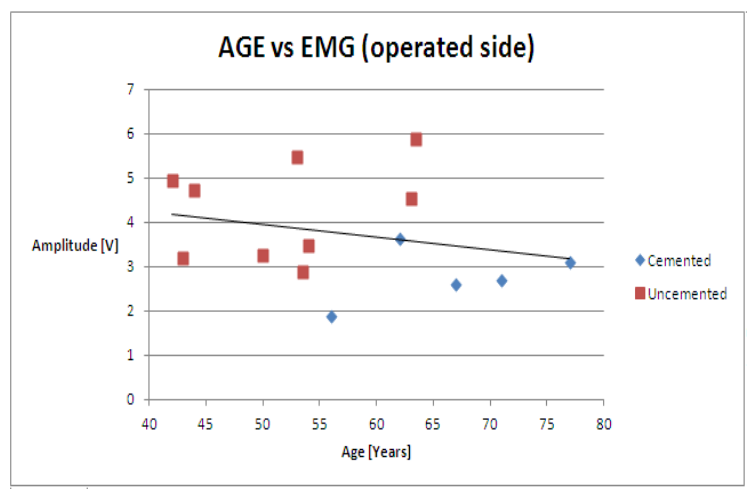

Fig 7 EMG amplitude of the Rectus Femoris sorted by implant technique and patient age. 
Gait Analysis, bone and muscle density in THA patients

European Journal Translational Myology - Basic Applied Myology 2012; 22 (4): 155-160

generally weaker. Figure 5 shows that this isn't always true, in fact BMD is, in several cases, above the trend line and higher compared with younger, non-cemented patients.

The pre-operative HU based density measurements of the Rectus Femoirs muscle of the operated leg also show a trend of decreasing with age. This is as expected, but as in the case of the BMD there are several cases of older patients that have higher HU compared to those that are younger and receive noncemented implant (Figure 6).

The post-operative EMG measurements on the Rectus Femoris of 14 patients follow the same trend as the BMD measurement and the muscle HU measurement. The amplitude of the muscles decreases with age, though some cases are above the trend line despite high age. Opposite to the other two measurements there are no cases of cemented patients that are above the trend line (Figure 7).

Preoperative measurements of bone- and muscle quality and muscle EMG evaluation are not a standard today, although it is commonly accepted that old individuals with low bone and muscle quality are good candidates for cemented THA. The results confirm the tendency of decreasing BMD with patient age as expected with the exception in several cases, where BMD was above the trend line and higher compared with young, non-cemented patients. The same goes for muscle HU evaluation of the Rectus Femoris of the operated leg where density decreases with age, but several cases of older and cemented patients are above the trend line. The preliminary data from EMG measurements of the Rectus Femoris of the operated leg show the same trend, decrease with age, but the older and cemented patients are never above the trend line. There is a possibility that the muscle $\mathrm{HU}$ measurements can be correlated with the EMG measurements, if that is possible it could be beneficial to the THA patient evaluation. But these are only preliminary results and the data would need further evaluation.

In the gait analysis, gait improves usually after THA but can be slight asymmetrical even after full recovery [12]. The results showed spatial parameters such as step length and stride length increase in most of the cases in the cemented implant group after 6 weeks and for both patient groups, an angle decrease was measured in the toe in/out parameter after 6 weeks.

The preliminary results based on various computational process that are able to quantitatively analyse THA patient's condition pre- and post-surgery are actually indicating very innovative methods and tools for the more correct selection of patients to cemented vs. non-cement type of surgery.

\section{Acknowledgements}

This work has been supported by the University Hospital Landspitali Scientific fund, the Health Technology Venue, Rannís and The Icelandic Student Innovation Fund.

Benedikt Magnússon is a 26 year old master student at Reykjavik University, Iceland in Biomedical Engineering. $\mathrm{He}$ is starting his second year of graduate studies and this work represents part of his master thesis. His research interests are medical modelling and clinical applications of medical models.

\section{Corresponding Author}

Paolo Gargiulo, Department of Science, Education and Innovation, Landspitali University Hospital and Department of Biomedical Engineering, University of Reykjavik, Iceland E-mail: paologar@landspitali.is

E-mails of co-authors:

(1) benediktm08@ru.is

(1) throsturp08@ru.is

(3) gigjamag@landspitali.is

(3) gretarh@landspitali.is

(4) jtriebel@gmail.com

$(4,5)$ halldor@landspitali.is

\section{References}

[1] Bishop NE, Ferguson S, Tepic S. Porosity Reduction in Bone Cement at the Cement-stem Interface. J Bone Joint Surg [Br] 1996;78-B:349356.

[2] GaitRite. Product: GaitRite. Available at: http://www.gaitrite.com/. Accessed in August 2012.

[3] Gargiulo P. Anthropometry of human muscle using segmentation techniques and 3D modeling. Handbook of Anthropometry: Physical Measures of Human Form in Health and Disease. Springer book 2012, Pages 324-354.

[4] Gargiulo P, Helgason T, Reynisson PJ, Helgason B, Kern H, Mayr W, Carraro U. Monitoring of muscle and bone recovery in spinal cord injury patients treated with electrical stimulation using three-dimensional imaging and segmentation techniques: Methodological assessment. Artif Organs. 2011; 35(3): 275-281.

[5] Hailer NP, Garellick G, Kärrholm J. Uncemented and cemented primary total hip arthroplasty in the Swedish Hip Arthroplasty Register, Acta Orthop 2010; 81(1): 34-41.

[6] Kine. Product: Kine Pro. Available at: http://kine.is/. Accessed in August 2012.

[7] Materialise. Product: MIMICS. Available at: http://www.materialise.com. Accessed in August 2012.

[8] Modus Medical Devices, Inc. QUASAR multipurpose body scanner. Available at: 
Gait Analysis, bone and muscle density in THA patients

European Journal Translational Myology - Basic Applied Myology 2012; 22 (4): 155-160

http://www.modusmed.com/quasar_mpbdyphntm. hhtm. Accessed in August 2012.

[9] Olsson E, Goldie I, Wykman A. Total hip replacement. A comparison between Cemented (Charnley) and Non-cemented (HP Garches) Fixation by clinical Assessment and objective Gait Analysis. Scandinavian J Rehab Med 18: 107-116, 1985.

[10] Taaffe DR, Cauley JA, Danielson M et al. Race and sex effects on the association between muscle strength, soft tissue, and bone mineral density in healthy elders: the health, aging, and body composition study. J Bone Miner Res 2001; 16: 1343-1352.
[11] van Loon CJ, de Waal Malefijt MC, Buma P, Verdonschot N, Veth RP. Femoral bone loss in total knee arthroplasty. Acta Orthop Belg 1999; 65(2): 154-163.

[12] Wykman A, Olsson E. Walking ability after total hip replacement. A comparison of gait analysis in unilateral and bilateral cases. J Bone Joint Surg Br 1992; 74(1): 53-56.

[13] Yamada H, Yoshihara Y, Henmi O, Morita M, Shiromoto Y, Kawano T, Kanaji A, Ando K, Nakagawa M, Kosaki N, Fukaya E. Cementless total hip replacement: past, present, and future. J Orthop Sci. 2009; 14(2): 228-241. 